\title{
A Novel Grid-Based Data Broadcasting Scheme for Wireless Sensor Networks
}

\author{
Arpit Bhushan Sharma ${ }^{1}$ Harshit Kargeti ${ }^{2 *}$ \\ 1 KIET Group of Institutions, Delhi-NCR, Ghaziabad, Uttar Pradesh, India 201206; arpit3043@gmail.com \\ 2 ABES Institute of Technology, NH 24, Delhi-NCR, Ghaziabad, Uttar Pradesh, India 201009; \\ hkargeti@gmail.com \\ * Correspondence: arpit3043@gmail.com; Tel.: (+918445726929)
}

\begin{abstract}
A run-of-the-mill remote sensor arrange comprises numerous vitality compelled sensor hubs that are arbitrarily sent is the sensor field. Vitality is one of the most significant parts of planning an information dispersal convention for applications, for example, war zone checking, living space observing, and so on. We present EGDD, an Energy-effective Grid-based Data Dissemination plot for arbitrarily conveyed remote sensor systems. The proposed conspire is vitality effective for dealing with both sink and source portability. EGDD depends on a virtual framework-based foundation for information scattering from various versatile sources to different portable sinks. The virtual lattice of square size is built by the main source showing up in the sensor field dependent on the remaining vitality of the sensor hubs. Moreover, we proposed an askew sending calculation for inquiry and information sending which guarantees that just a single spread hub advances the question and information at once. In EGDD, substitute scattering hubs are chosen ahead of time during the lattice development procedure and the least limit vitality level is characterized for spread hubs. When the vitality of a dispersal hub arrives at the base edge esteem, it is supplanted by another spread hub. The reenactment results show that the proposed EGDD conspire is more vitality proficient when contrasted with other information scattering conventions.
\end{abstract}

Keywords: dissemination node; reference coordinates; crossing point; residual energy; sink manager

\section{Introduction}

Late advances in microelectronic-mechanical frameworks (MEMS), VLSI, microchip, and remote correspondence innovations have empowered the structure and sending of enormous scope sensor systems. Numerous sensor hubs are haphazardly conveyed over an immense field to self-arrange an enormous scope remote sensor organize [1-4]. These sensors are regularly little, minimal effort, multipractical and battery-controlled gadgets. In this paper, we have proposed an information scattering plan to take care of the issue of versatile and vitality productive information dispersal in a haphazardly sent sensor organize from different sources to numerous, compact sinks. In the following work, a corresponding source insinuates a sensor center point that distinguishes the event in its neighborhood and makes identifying data to report about the event to the sink or base station. Sink is a customer which accumulates the data reports from the sensor organize. Both the sink and the event may be flexible and the quantity of events and that of the sinks may vary after some time. Consider the case of a sensor arrange for front line observing where the number of warriors can be considered as versatile sinks and a rival tank can be considered as a portable occasion (Figure 1). The sensors encompassing a tank identify it and team up among themselves to total information, and one of them produces an information report [5]. The moving warriors gather these information reports from the sensor hubs. In 
the following paper, we have put forward a framework-driven information dispersal plot about haphazardly sent, consistently appropriated sensor systems in which sensors are heterogeneous as far as vitality (battery level). We acknowledge that each sensor center point thinks about its zone coordinate in bi-dimensional sensor area (for example, using GPS indicators). An implicit structure is created by the essential source appearing in the sensor field and all other coming about appearance of sources is served by a comparative cross section establishment. The sensor hubs at the edges of the square measured virtual framework are known as dispersal hubs. Question and information are sent through the dispersal hubs just and every single other sensor doesn't take an interest in inquiry and information sent. We have proposed a system model to deal with the irregular organization of sensors during the framework development process which comprises a normal zone to choose a scattering hub at (or nearest to) the network crossing focuses. A base limit vitality level is characterized for the spread hubs and when the leftover vitality of a dispersal hub reaches that level, it is supplanted by another scattering hub. Further, we have proposed an askew sending calculation for question and information sending which guarantees the inquiry/information transmission through the most limited conceivable way in the system and guarantees that just a single sensor hub transmits question/information at once. Sink portability is dealt with by utilizing sink supervisor and capacity ability of spread hubs is utilized for taking care of source versatility.

The principal commitments of this paper are featured as follows.

- Develop an information spread plan to diminish the number of transmissions and inquiry flooding costs.

- Design a vitality effective information scattering plan that can transmit parcel with the least postponement.

- Propose a matrix development conspire for arbitrarily sent sensor arrange where the sensors may not be available at the specific evaluated position during framework development.

- Propose systems to understand the sink and source versatility issues.

- Propose the askew sending calculation for question/information sending through the briefest conceivable way in the sensor organize.

The remainder of the paper is composed as follows. Section 2 presents a short presentation of a portion of the current network-based information dispersal plans. We present the system model, suppositions, and proposed EGDD conspire in Section 3, and assess EGDD execution in Section 4. At long last, Section 5 sums up this paper with a conclusion.

\section{Related Work}

Several lattice-based data dissemination protocols has been contemplated over the course of years for handling sink mobility in WSNs. Virtual matrix based plans are extremely well known in light of the fact that the utilization of a little virtual framework may not just decrease the vitality cost of information scattering yet it likewise may diminish the convention repetition, dependability, and strength as it focuses the traffic over a little structure, instigating blockage, sudden passing of hubs (i.e., abused hubs), and the presence of basic hubs. In 2005, Luo et al. [6] proposed a two-level information dispersal model for enormous scope remote sensor systems. TTDD is a two-level 
information spread plan in which a virtual matrix of square-size cells is developed by each source showing up in the sensor field. In TTDD, upon the identification of another occasion, the source hub proactively manufactures a virtual network structure. An inquiry from a sink hub is sent through the upstream hubs just to arrive at the source hub. In 2008, Sharma et al. [7] proposed a matrix based information dispersal plot (GBDD) that utilizes a double radio method of sensor hubs to choose the cell size of a network for example high and low force radio reaches are utilized to choose the size of the square estimated cell. Not at all like TTDD, where each new occasion (for example source hubs detecting occasion) starts matrix development, this methodology builds a lattice just when the sink first time shows up in the sensor field which stays substantial for an exceptionally huge term. GBDD misuses area mindfulness and double radio method of sensor hubs to manufacture matrix structure over the whole sensor field. In 2010, Soochang et al. [8] proposed an information dispersal plot that follows the distribute/buy in worldview. Referee develops an autonomous lattice structure to which source hubs and sinks are namelessly and non-concurrently associated and gives information multicasting by the structure for the space decoupling. Judge likewise oversees capacity and planning of information and questions on the structure for time decoupling. Source hubs, sinks, and general sensor hubs that do not include the association of the structure don't partake with control motioning for information interworking for the synchronization decoupling. Judge utilizes the dynamic impression tying to help neighborhood portability of sinks in a framework cell. Information scattering to various versatile sinks in enormous scope WSNs is a significant examination field as it requires a great deal of upgrades in the virtual lattice development procedure, information and inquiry sending and taking care of spread hub disappointment.

\section{Proposed Data Dissemination Scheme}

We have proposed a vitality effective matrix-based information scattering plan (EGDD). In the proposed plot, just a single virtual lattice is developed and all the sources showing up in the sensor field utilize the current matrix foundation. Information is forwarded to the hub utilizing the most limited way in the framework employing scattering hubs. Sink and occasion versatility (otherwise called sink and source portability) carry new difficulties to huge scope remote sensor systems. We propose EGDD, an Energy-Competent Grid-based Data propogation convention, to address both the source and sink versatility and the vitality protection issues.

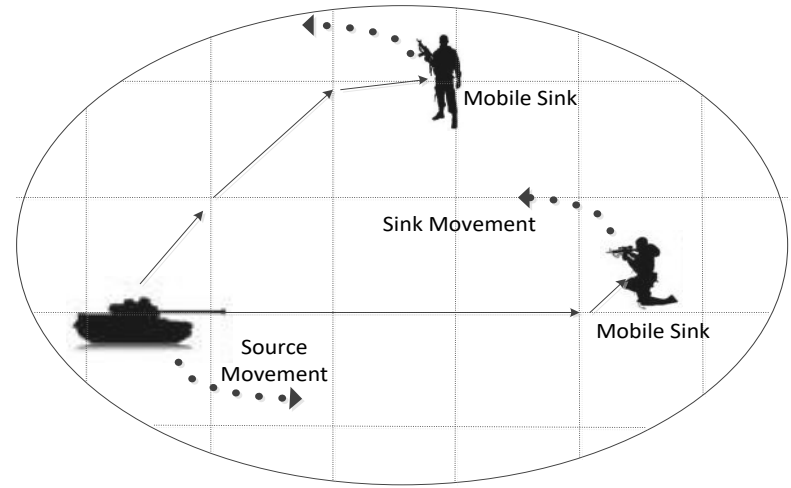

Figure 1: Soldiers detect the opponent tank using a sensor network.

\subsection{Design Principle}

A distant sensor core is considered as a tool developed by three fundamental units, the microcontroller unit (MCU), the hand-set unit (TCR), and sensor board (SB). Each of these units will 
absorbed a particular degree of vitality while working. The vitality absorbed by a distant sensor core is communicated as

$$
E_{\text {tot }}=E_{\text {comp }}+E_{\text {trans }}+E_{\text {sens }}
$$

Etrans is the vitality devoured in transmitting and getting the information and is corresponding to the separation of transmission. In this way, Energy utilization can be diminished by decreasing the number of transmissions and the separation of transmission. Inquiry and information sending is done through the most limited way in the virtual network foundation. Every dispersal hub is equipped for speaking with all its neighboring spread hubs including the scattering hubs found askew. The remaining vitality of sensor hubs is additionally thought of while choosing the scattering hub to dodge visit spread hub disappointment. The System Assumptions are as following utilized in this paper to organize displaying:

- Every sensor hub knows about its area arranges in a X-Y Plane of sensor dimensional field (e.g using GPS indicators).

- The Sensor nodes are static and skillful of doing computation and storing some information regarding the neighboring nodes.

- Every sensor node is mindful of its extra energy level.

\subsection{Network Model}

In the proposed information scattering plan, a normal zone with sweep $d$ is developed and cell size is dictated by utilizing the radio scope of sensors (R) and range $d$ of the anticipated zone. The expected zone is utilized for dealing with the vulnerability in the sensor hub area. The dispersal hub is chosen among all the sensor hubs lying in the normal zone. Along these lines, the sensor hub with the most extreme leftover vitality is chosen as the dispersal hub (Figure 2).

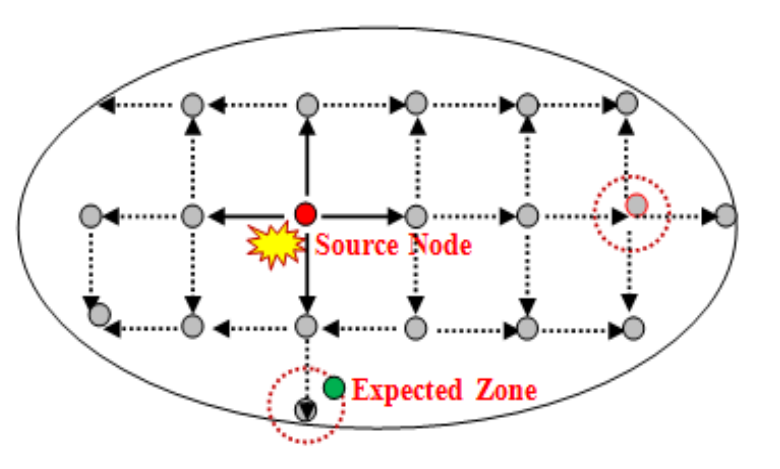

Figure 2: Network Model

\subsection{Grid Construction}

In the proposed information scattering plan, the network development course is initiated from the source just when there is no substantial lattice present in the sensor field. At the point when an occasion or improvement shows up in the sensor field, the sensor hub closest to the occasion goes about as a sourcing hub and starts the network development process by creating a question for lattice development. The inquiry comprises of the directions of the evaluated crossing point, cell size $\alpha$, and span of anticipated in zone $d$. 


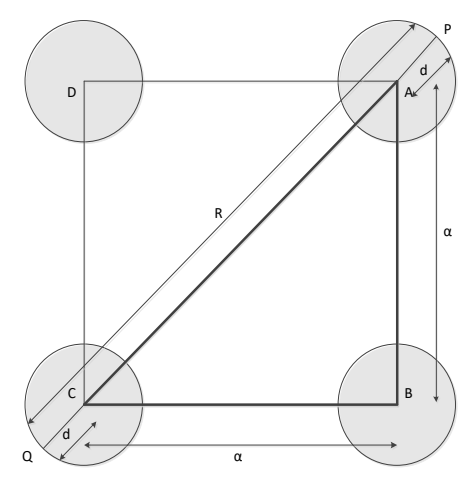

Figure 3: Determining the Cell size of the grid

- Finding the cell magnitude $\alpha$ :

Cell magnitude is dictated by utilizing the radio range $\mathrm{R}$ of sensor hubs and the constraining removed. We can decide the cell magnitude $\alpha$ with the assistance of figure 3 .

Here, the figure 3 shows that

The cell size is,

$$
\mathrm{BC}=\mathrm{AD}=\mathrm{CD}=\mathrm{AB}=\alpha
$$

The range of excepted zone,

$\mathrm{AP}=\mathrm{CQ}=\mathrm{d}$

In this manner, we can apply Pythagoras Theorem in triangle $\mathrm{ABC}$,

The distance $\mathrm{AC}=\alpha \sqrt{2}$

The radio scope of the hub ought to be equivalents to $\mathrm{PQ}$,

$\Rightarrow \mathrm{PQ}=\mathrm{R}$

Since,

$$
\begin{aligned}
& \mathrm{AP}+\mathrm{AC}+\mathrm{CQ}=\mathrm{R} \\
& \Rightarrow \mathrm{d}+\alpha+\mathrm{d}=\mathrm{R} \\
& \Rightarrow \alpha+2 \mathrm{~d}=\mathrm{R} \\
& \Rightarrow \alpha=\mathrm{R}-2 \mathrm{~d} \\
& \alpha=(\mathrm{R}-2 \mathrm{~d}) / \sqrt{ } 2
\end{aligned}
$$

From figure 3, it is very certain that every sensor hub is inside the immediate radio scope of one another hub for example a scattering hub at any intersection purpose of the framework cell can discuss straightforwardly with all other dispersal hubs in the area.

- Finding the radius $\mathrm{d}$ of the expected zone:

We would build up a circular expected zone with the radius $\mathrm{d}$.

$\pi \mathrm{d} 2$ will be area of expected zone.

Let there be $\mathrm{n}$ sensor hubs disposed in sensor area and the field of region is A. Then,

Area of finding out one sensor hub $=$ total area/ total numbers of sensor

$$
\pi \mathrm{d}^{2}=\mathrm{A} / \mathrm{n}
$$


For a dissemination node $i$ located at $\left(\mathrm{X}_{\mathrm{i}}, \mathrm{Yi}\right)$, crossing points $\left(\mathrm{X}_{\mathrm{j}}, \mathrm{Y}_{\mathrm{j}}\right)$ are calculated as

$$
\left\{\mathrm{X}_{\mathrm{j}}=\mathrm{X}_{\mathrm{i}}+\alpha, \mathrm{Y}_{\mathrm{j}}=\mathrm{Y}_{\mathrm{i}}+\alpha\right\}
$$

On getting the inquiry, every sensor hub computes its separation (di) from the evaluated crossing point. If the separation is not as much as $\mathrm{d}$, at that point the sensor hub ascertains its Mean Factor Mi and answer with $\mathrm{Mi}$, in any case, dispose of the question. Therefore, the sender will get the mean estimations of every sensor hub existing in the normal zone. Presently the sensor hubs are masterminded in plummeting requests of their mean qualities and the hub with a most noteworthy mean worth is chosen as the scattering hub. A similar procedure is rehashed until the whole sensor field is secured by the matrix. In the event of scattering hub disappointment, the second hub from the rundown is chosen as the dispersal hub. Therefore, there is no need to question flooding for taking care of spread hub disappointment.

\subsection{Dissemination Node Selection}

The sensor hub with the most elevated mean worth is chosen as the scattering hub. Mean Value is characterized as the augmentation of the Distance Factor (Di) with Residual Energy (Ei).

\subsubsection{Calculating Distance Factor $D_{i}$}

$$
D_{i}=\left|d_{i}-d\right| / d
$$

Where, $d_{i}$ is the distance of sensor hub from intersection point and the radius of anticipated zone is $\mathrm{d}$.

\subsubsection{Calculating Mean Value Mi:}

$$
M_{i}=D_{i} \times E_{i}
$$

Where the remaining energy of sensor node $i$ is $\mathrm{E}$.

A rundown of all these sensor hubs is kept up in the slipping request of their mean qualities and is utilized for taking care of spread hub disappointment. Therefore, the proposed conspire not just chooses the best hub as a dispersal hub yet also keeps up a rundown of exchange spread hubs.

\subsection{Forwarding of data and query}

We devise a question sending plan (Figure 5) regarding information spread to WSN in compact sinks. Not at all like TTDD and GBDD, the proposed plot does not advance inquiry at the edges of the lattice cell rather it depends on sending the question through the briefest way among source and sink. In the proposed plot, just the dispersal hub which is nearest to the source advances the inquiry. We accept that every scattering hub knows about its area (arranges). Exactly when a sink needs to propel the inquiry to a source, it makes a request pack with Source Sequence Number, Sink id and encourages that source dispersal center point (Xs, Ys). By and by it progresses the request package to all of its neighbors. At the point when the inquiry is gotten by a spread center point, it is sent to the data source. Data is sent to the sinks through the contrary route followed by the inquiry. In the event that a dispersing center had gotten requests from different neighboring center points, it sends a data copy to all of them. At the point when the data appears at a sink's close by spread center, heading sending [2] is used to furthermore move the data to sink. Figure 4 shows the methods followed by the proposed EGDD scheme for data sending from any event source to the interested sink. 


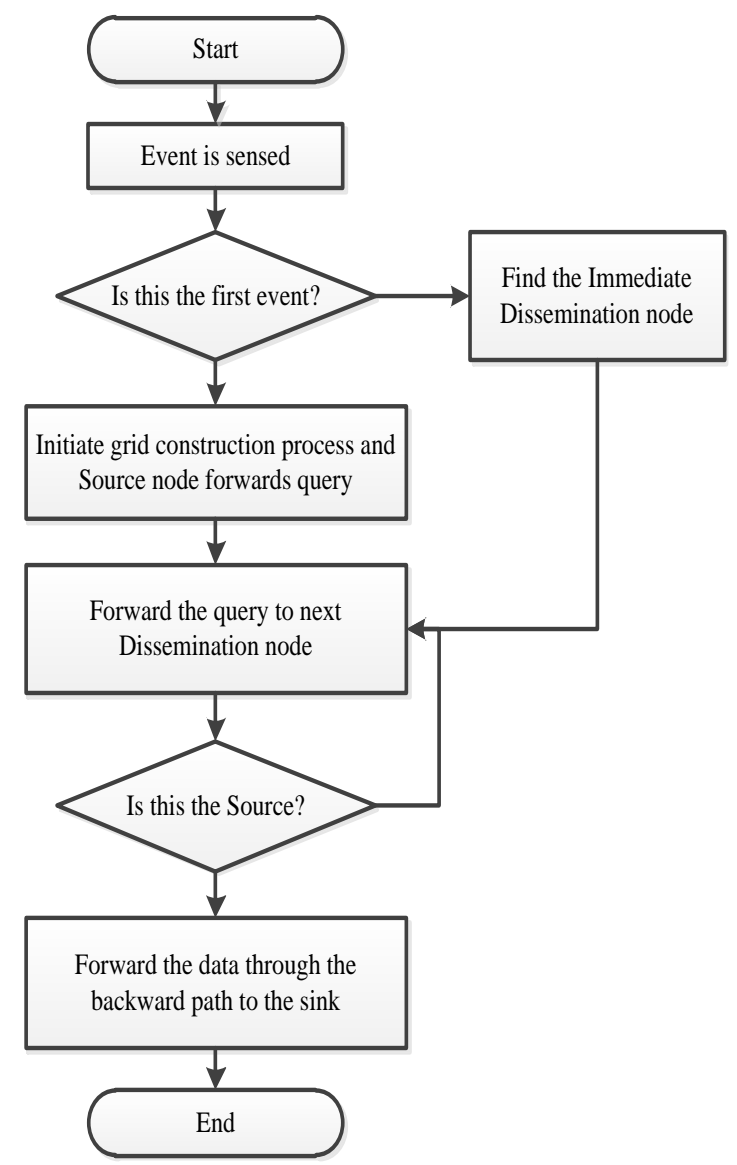

Figure 4: Flow Chart for EGDD

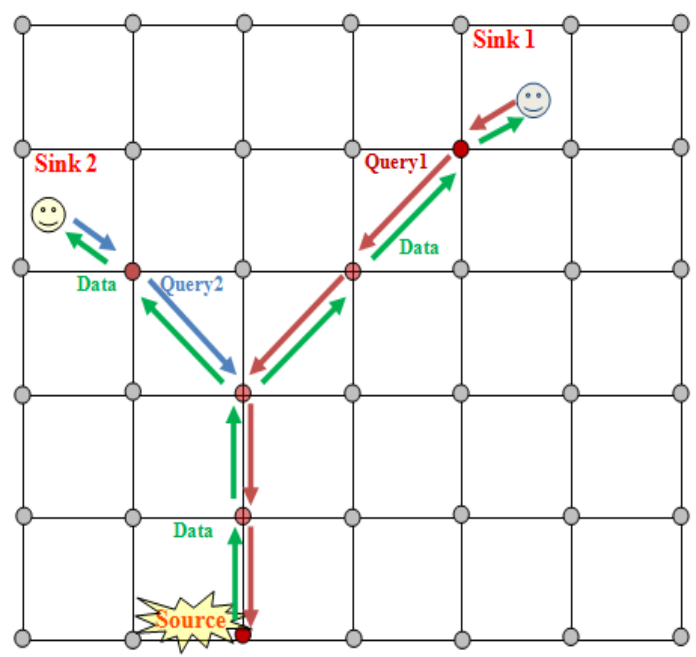

Figure 5: Query and Data Forwarding

\subsection{Query and Data Forwarding with Multiple Sources}

Exactly when the source appears in sensor field, this uses the current cross section for data sending. Every source has an outstanding source identification as the region headings of the snappy dissipating center point (IDN) is used as the source id. The event source forms a data announcement with its remarkable source id and the sink fascinated by that event progresses the inquiry using the current grid in the sensor field. Data is sent from various sources to different sinks using the backward route crossed by the inquiry pack. Request and data sending have showed up in figure 6 . 


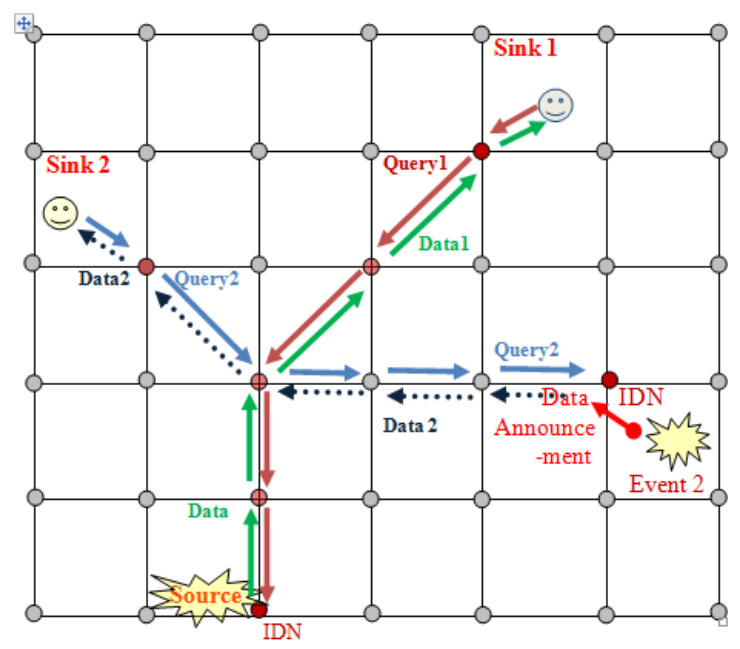

Figure 6: Data Forwarding from Multiple Sources

\subsection{Handling Multiple Sinks and their Movements}

In WSNs, Data scattering is required from conceivably various occasion sources to possibly different sinks. There might be various sinks intrigued by a similar occasion source or various sinks keen on various occasions. At the point when a dispersal hub gets various questions for similar information from various sinks, it advances just one inquiry towards the source and keep the sink identification of considerable number of sinks from where the question for similar information is gotten. At the point when Data is gotten at this scattering hub, copy duplicates of information are produced and sent to all the sinks from where the inquiry was gotten. While If the questions from various sinks for the diverse occasion are gotten, at that point all the inquiries are sent to individual spread hubs. The information is sent to the sink through the regressive way followed by the question. Sink versatility is one of the significant difficulties in the information scattering process. A versatile sink, for example, a fighter can move starting with one spot then onto the next in the wake of sending the question for some occasion of intrigue. A sink may move into any of four neighboring cells around its IDN. For this situation, there is no overhead of following sink versatility as the IDN can straightforwardly advance the information to the moving sink since all the four cells are in the immediate transmission scope of the IDN. Be that as it may, when a sink moves from these four cells, it is required to follow the sink development.

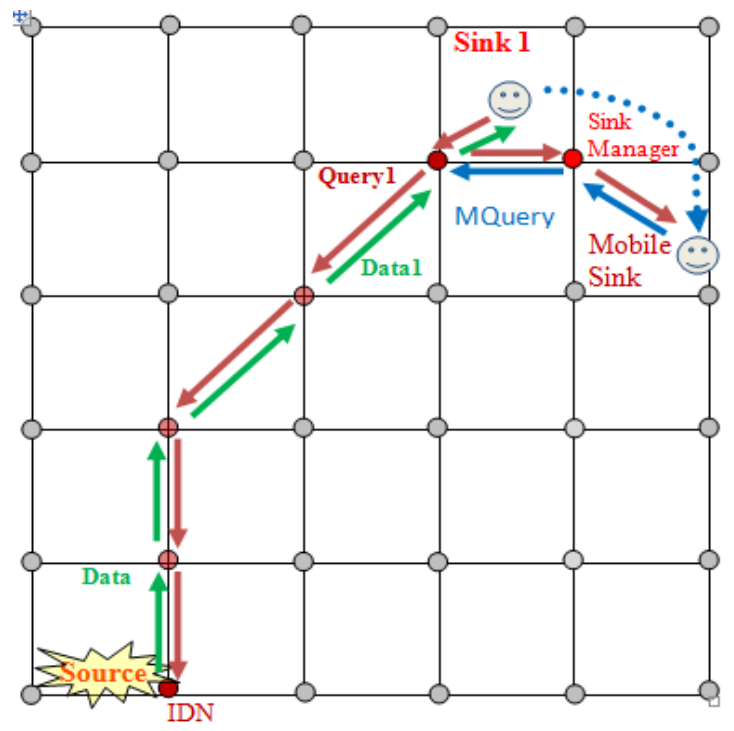


Figure 7: Handling Sink movement using Sink Manager

A sink director (SM) is utilized for taking care of sink versatility. A sink chief is a scattering hub which is regular to past cell and current cell of a sink and farthest to the past IDN/sink director. At the point when a sink moves to start with one cell then onto the next nearby cell, a sink chief is chosen, and the past IDN/sink administrator is educated about the area of the current sink director. A similar procedure is rehashed each time when a sink maneuver from one cell to another cell region. The information is forwarded back to the current sink administrator utilizing the area data of the sink chief (as appeared in figure 7). Along these lines, rather than choosing another sensor of taking care of sink development, EGDD utilizes the scattering hubs.

\subsection{Handling Mobile Events}

A sink director (SM) is utilized for taking care of sink versatility. A sink administrator is a spread hub which is regular to a past cell and a current cell of a sink and farthest to a past IDN/sink director. At the point when a sink moves to start with one cell then onto the next adjoining cell, a sink supervisor is chosen, and the past IDN/sink chief is educated about the area of the current sink administrator. A similar procedure is rehashed each time when a sink moves from one cell to another cell territory. The information is sent back to the current sink director utilizing the area data of the sink chief (as appeared in figure 7). Accordingly, rather than choosing another sensor of taking care of sink development, EGDD utilizes the scattering hubs. In the proposed EGDD plot, we utilize the capacity ability of the sensor hubs for dealing with the occasion portability. Portable occasions, for example, tanks, vehicles, or creatures can be followed by putting away the detected data by source and sitting tight for quite a while. At the point when a sourcing hub identifies that the occasion has versatility, it stores the detected information, sits tight for an edge time, and makes an information declaration if it has not gotten some other information report from neighboring hubs. If the occasion moves to another area and detected by another source hub it will likewise hang tight for the limited time.

On the off chance that during this time it gets the information declaration (information report), it doesn't make information declaration rather it advances the information report about the occasion to the dispersal hub from where it has gotten the information declaration. The source hub totals the information and updates the data about the source. Such a collection of information about the portable occasion can decrease the flooding of an enormous number of information reports and declarations in the system

\section{Performance Evaluation}

In this segment, we assess the exhibition of the proposed EGDD plan and contrast it and TTDD. Initially, we characterize reproduction boundaries and execution measurements and afterward observe the impact of different factors, for example, the quantity of sources, quantity of sinks and sink speed on the exhibition of the proposed EGDD approach.

\subsection{Simulation Parameters and Performance Metrics}

We actualize EGDD convention in $\mathrm{OMNeT++}$ recreation structure. We utilize indistinguishable boundaries for the reproduction from utilized by TTDD. We use the IEEE 802.11DCF MAC convention. 200 sensors are arbitrarily conveyed in a $2000 \times 2000 \mathrm{~m} 2$ sensor field. Every reproduction run goes on for 200 seconds. The size of the inquiry bundle is 36 Bytes and that of information parcel 
is 64 Bytes. A sensor hubs default power utilization rates are set as: transmitting power $=0.66 \mathrm{~W}$, getting power $=0.395 \mathrm{~W}$ and sitting force $=0.35 \mathrm{~W}$. We utilize a limit of 5 sources and 5 sinks for the recreation. We utilize three execution measurements to assess the presence of EGDD. The absolute vitality utilization is characterized as the all-out vitality devoured in transmitting and accepting in the system. Vitality devoured by the sensors out of gear state is not checked since it relies to a great extent upon the information age span and has no connection with vitality productivity. The normal deferral is characterized as the normal time expended between the transmission of a parcel from the source and gathering of a similar bundle by the sink for all source-sink sets. The achievement rate is characterized as the proportion of the quantity of effectively got information parcels at the sink to a complete quantity of information bundles created by the source, arrived at the midpoint of for all source-sink sets.

\subsection{Effect of Number of Sources and Sinks on Total Energy Consumption}

Figures 8(a) and 8(b) show the complete vitality utilization by EGDD and TTDD separately with a changing quantity of sources and sinks. Figure 8(a) denotes that for each bend, the absolute vitality utilization increments step by step as the quantity of sinks increments because as the quantity of sinks expands, the number of transmissions likewise increments. The all-out vitality utilization doesn't increment directly because as the quantity of sinks expands the questions from various sinks for a similar source can likewise be converged at some dispersal hub. Be that as it may, for a few sinks, when we increment the number of sources, absolute vitality utilization increments directly because the complete number of questions and information bundles expands relatively and brings about a straight increment in all-out vitality utilization. From figure 8(a) and 8(b), all-out vitality utilization in EGDD is around $40-45 \%$ not exactly TTDD.

\subsection{Effect of Number of Sources and Sinks on Delay}

The effect of differing quantity of sinks and sources on deferring when EGDD and TTDD are utilized is appeared in figure 9(a) and 9(b) separately. The deferral in EGDD is lower when contrasted with TTDD because in EGDD the inquiry and information bundles follow the slanting way generally. From figures 9(a) and 9(b), we can presume that EGDD appears at a 35\% improvement in normal deferral registered overall source-sink sets for an information bundle to reach from source hub to sink.

\subsection{Effect of Sink Mobility}

Figure 10 denotes the aftermath of sink speed on the progress rate. In the default reproduction setting, we differ the sinks' most extreme speed from $0 \mathrm{~m} / \mathrm{sec}$ to $20 \mathrm{~m} / \mathrm{sec}$ and assess the effect of this variety in progress rate. The achievement rate for EGDD is somewhat superior then TTDD as appeared in figure 8 . The achievement rate is higher (around 89\%) for low sink speed though it is around $85 \%$ for a high sink speed of $20 \mathrm{~m} / \mathrm{sec}$. 


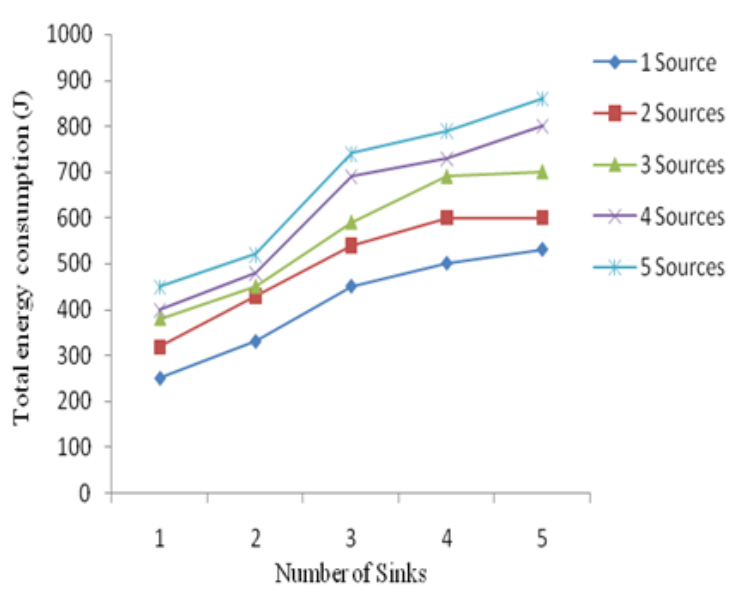

Figure 8(a). The plot between quantity of Total energy consumption and Sinks for EGDD

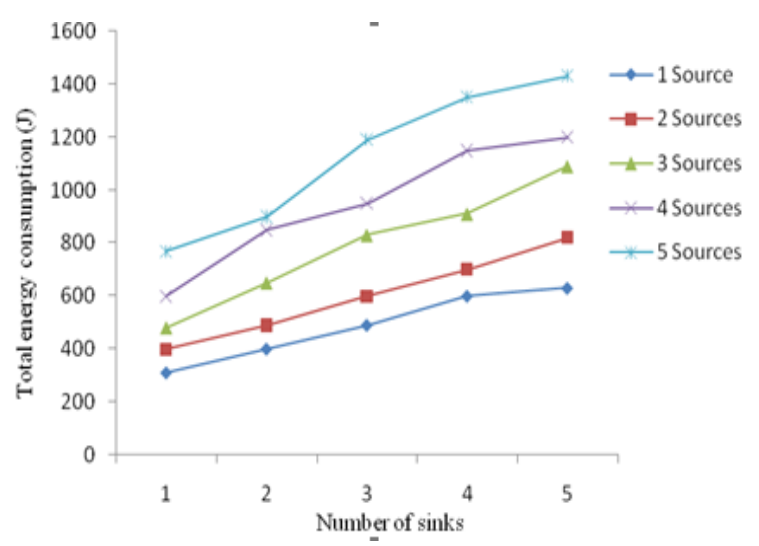

Figure 8(b): Plot between quantity of Total energy consumption and Sinks for TTDD

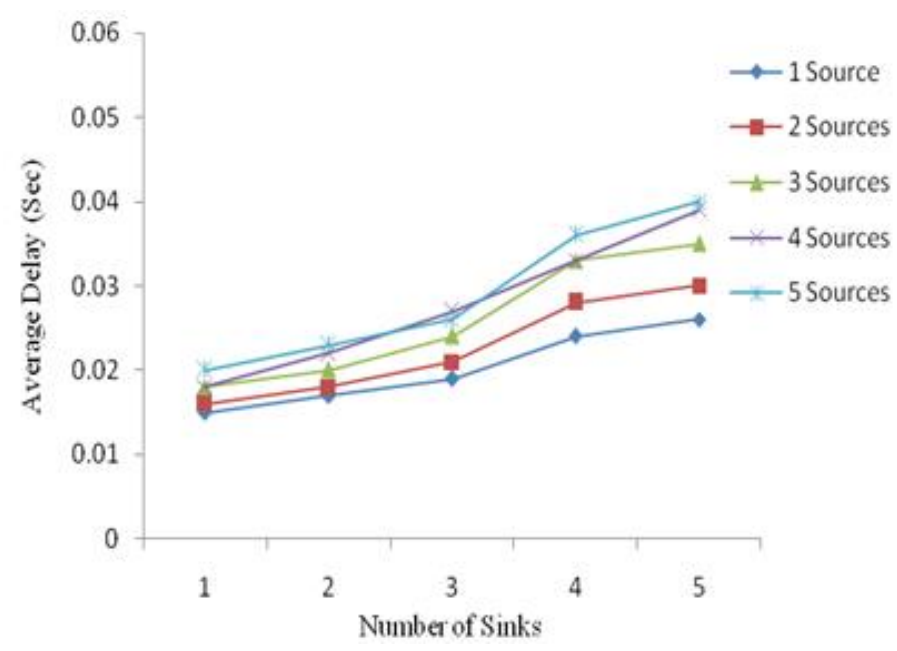

Figure 9(a): Plot between Number of Sinks and Average Delay for EGDD 


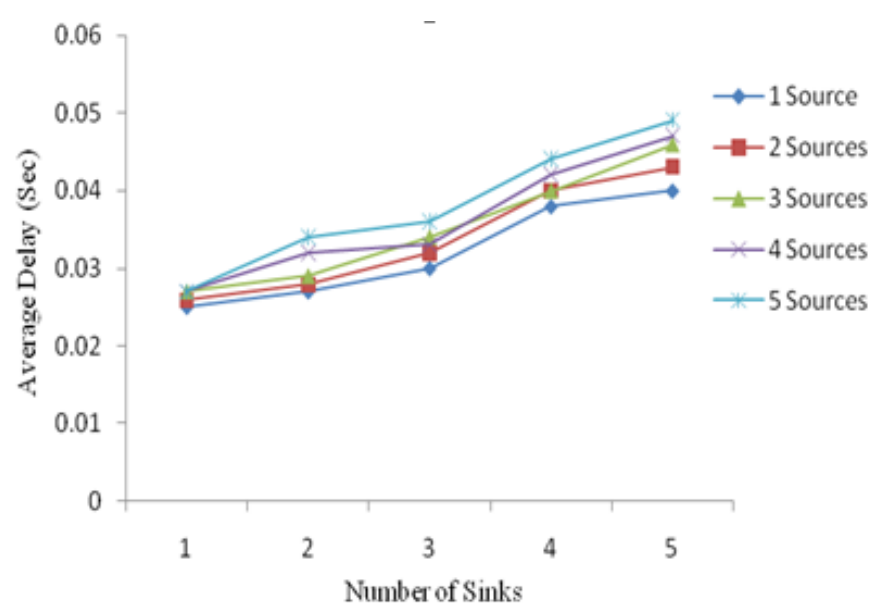

Figure 9(b): Plot between Number of Sinks and Average Delay for TTDD

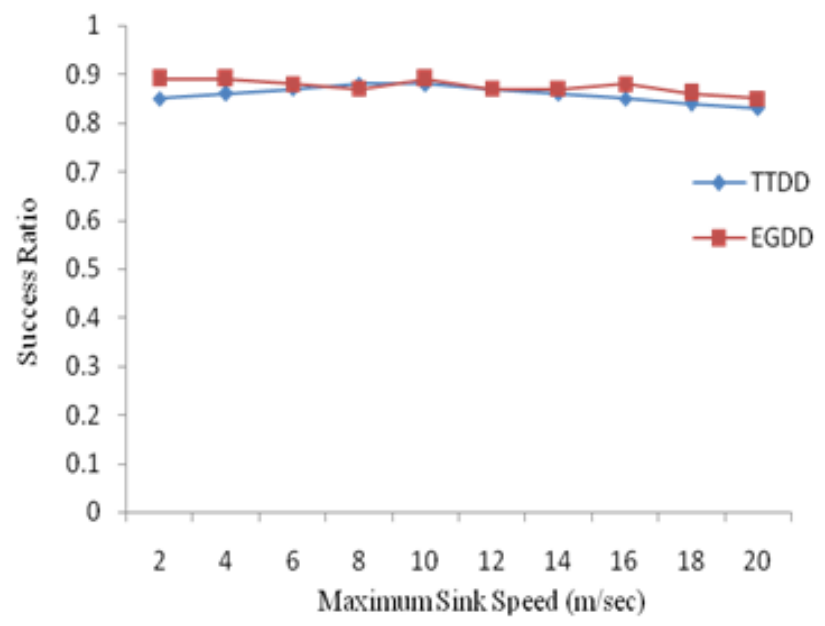

Figure 10: Plot between Success Rate Vs Maximum Sink Speed

\section{Conclusions}

In this paper, we have proposed a virtual framework based information spread plan for haphazardly conveyed WSNs. EGDD is a novel information scattering plan which utilizes a solitary virtual matrix foundation for question and information sending from possibly different portable sources to conceivably various versatile sinks. Dispersal hubs are chosen dependent on their remaining vitality and compelling good ways from intersection focuses. The proposed EGDD conspire is vitality effective and hearty as it diminishes the vitality utilization in question and information sending and spread hubs are supplanted before consumption of the vitality. The proposed to arrange model guarantees that every scattering hub can straightforwardly speak with each of the 8 neighboring spread hubs in one-jump. Moreover, we proposed a slanting sending calculation to guarantee that question and information are sent through the most limited way between each source-sink sets and just a single scattering hub advances the inquiry/information at once. Sink portability is taken care of utilizing sink chiefs while occasion versatility is dealt with by utilizing the capacity ability of source hubs. The proposed EGDD plot is more vitality productive than TTDD for different sinks and numerous sources. Simultaneously, EGDD can keep up a lower normal deferral because of fewer impacts. Reproduction results show huge enhancements in the exhibition of EGDD as contrasted and TTDD.

\section{Forthcoming Work}

In the future, we intend to misuse the proposed information scattering plan for non-consistently circulated WSNs. We might likewise want to misuse the plan for conceiving a successful in-arrange information total plan and store the board plot. 


\section{References}

1. I.F. Akyildiz, W. Su, Y. Sankarasubramaniam, and E. Cayirci, "A survey on sensor networks," IEEE Communications Magazine, Vol. 40, pp. 102-114, Aug. 2002.

2. S. Tilak, N. B. Abu-Ghazaleh, and W. Heinzelman, "A taxonomy of wireless micro-sensor network models," ACM Mobile Computing and Communications Review, Vol. 6, Iss. 2, Apr. 2002, pp. 28-36, IEEE Transactions on Consumer Electronics, Vol. 56, No. 3, August 2010.

3. J.N. Al-Karaki and A.E. Kamal, "Routing techniques in wireless sensor networks: a survey," IEEE Wireless Communications, Vol. 11, Iss. 6, pp. 6-28, Dec. 2004.

4. H. Karl and A. Willing, "Protocol and Architecture for Wireless Sensor Networks," John Wiley \& Sons, Inc., 2005.

5. F. Ye, S. Lu, and L. Zhang, GRAdient Broadcast: A robust, longlived large sensor network (2001), http://irl.cs.ucla.edu/papers/grab-techreport.ps.

6. Haiyun Luo, Fan Ye, Jerry Cheng, Songwu Lu, Lixia Zhang, "TTDD: two-tier data dissemination in large-scale wireless sensor networks", Kluwer Academic Publishers' Wireless Networks, vol. 11 , issue 1-2, pp.161-175, January 2005.

7. T.P. Sharma, R.C. Joshi, Manoj Misra, “GBDD: Grid-Based Data Dissemination in Wireless Sensor Networks" Advanced Computing and Communications, 2008. ADCOM 2008, pp 234 - 240, 2008.

8. Soochang Park, Euisin Lee, Fucai Yu, and Sang-Ha Kim, "Scalable and Robust Data Dissemination in Large-scale Wireless Sensor Networks", IEEE transaction on Consumer Electronics, Vol. 56, No. 3, August 2010.

9. "Data Broadcasting Scheme for Wireless Sensor Networks - Google Scholar." https://scholar.google.com/scholar?oi=gsb95\&q=Data Broadcasting Scheme for Wireless Sensor Networks\&lookup=0\&hl=en (accessed Jul. 13, 2020).

10. O. Delgado-Mohatar, A. Fúster-Sabater, J. S.-A. H. Networks, and undefined 2011, "A lightweight authentication scheme for wireless sensor networks," Elsevier, Accessed: Jul. 13, 2020. [Online]. Available: https://www.sciencedirect.com/science/article/pii/S1570870510001307.

11. P. Havinga, “A Dynamic Data Aggregation Scheme for Wireless Sensor Networks,” 2003. Accessed:Jul. 13, 2020. [Online]. Available: http://www.cs.utwente.nl/ supriyo.

12. J. Li and G. Y. Lazarou, "A bit-map-assisted energy-efficient MAC scheme for wireless sensor networks," in Third International Symposium on Information Processing in Sensor Networks, IPSN 2004, 2004, pp. 55-60, DOI: 10.1145/984622.984631.

13. J. Li, X. Jiang, I. L.-J. of Sensors, and undefined 2014, “Energy balance routing algorithm based on virtual MIMO scheme for wireless sensor networks," hindawi.com, Accessed: Jul. 13, 2020. [Online]. Available: https://www.hindawi.com/journals/js/2014/589249/abs/.

14. C. O.-I. S. Journal and undefined 2011, “A localization scheme for wireless sensor networks using mobile anchors with directional antennas," ieeexplore.ieee.org, Accessed: Jul. 13, 2020. [Online]. Available: https://ieeexplore.ieee.org/abstract/document/5704171/.

15. W. Du, J. Deng, Y. Han, ... S. C.-I. I., and undefined 2004, “A key management scheme for wireless sensor networks using deployment knowledge," ieeexplore.ieee.org, Accessed: Jul. 13, 2020. [Online]. Available: https://ieeexplore.ieee.org/abstract/document/1354530/.

16. S. X. Wu, Q. Li, and A. M. So, "Rank-Two Beamforming and Stochastic Beamforming for MISO Physical-Layer," IEEE Signal Processing Letters, vol. 22, no. 10, pp. 1614-1618, 2015, DOI: 10.1109/LSP.2015.2416258.

17. S. M. Chang, S. Shieh, W. W. Lin, and C. M. Hsieh, "An efficient broadcast authentication scheme in wireless sensor networks," in Proceedings of the 2006 ACM Symposium on Information, Computer, and Communications Security, ASIACCS '06, 2006, vol. 2006, pp. 311-320, DOI: $10.1145 / 1128817.1128864$

18. M. Ye, C. Li, G. Chen, J. W.-P. 2005. 24th IEEE, and undefined 2005, "EECS: an energy-efficient clustering scheme in wireless sensor networks," ieeexplore.ieee.org, Accessed: Jul. 13, 2020. [Online]. Available: https://ieeexplore.ieee.org/abstract/document/1460630/.

19. D. Kumar, T. Aseri, R. P. communications, and undefined 2009, "EEHC: Energy efficient heterogeneous clustered scheme for wireless sensor networks," Elsevier, Accessed: Jul. 13, 2020. [Online]. Available: https://www.sciencedirect.com/science/article/pii/S0140366408006087. 